\title{
Ileal Lipoma: A Rare Lead Point for Adult lleocolic Intussusception
}

\author{
Adedire Adenuga \\ University of llorin Teaching Hospital, Ilorin, Kwara State, Nigeria \\ Correspondence to: Dr. Adenuga Adedire, PO Box 23431, Nigeria; email: timiadenuga@gmail.com
}

\section{Summary}

Intussusception is the telescoping of a segment of the gastrointestinal tract within the lumen of an adjacent segment. Adult intussusceptions are rare, with ileal lipoma responsible for only $6 \%$ of cases. Clinically, adult intussusception remains an elusive diagnosis. The triad of abdominal pain, vomiting and passage of bloody stool is not frequently seen in adults. Abdominal computed tomography is the gold standard for identifying ileal lipoma as the lead point of an adult intussusception. Definitive management is by surgical excision of the involved segment. This is a case report of a 38-yearold female with intussusception with a 5 -cm subserosal ileal lipoma as the lead point. This is the first (to the best of our

\section{Introduction}

Intussusception is defined as telescoping of a segment of the gastrointestinal tract within the lumen of an adjacent segment. Clinically, it is often misdiagnosed in adults and taken for more common causes of acute abdomen such as appendiceal pathology, gastrointestinal malignancy, and enteritis (1). Adult intussusception represents $5 \%$ of all cases of intussusception and accounts for $1-5 \%$ of intestinal obstructions in adults (2, 3 ). In contrast to intussusception occurring in children, adult intussusceptions have a pathologic lead point in up to $90 \%$ of cases (4).

Intestinal lipoma as a cause of intussusception is seen in only $6 \%$ of patients with intussusception (5). Intestinal lipoma usually presents with obstruction, though it may also present as gastrointestinal bleeding or diarrhea $(2,6)$. This is a report of a rare case of ileal lipoma causing an ileocolic intussusception requiring emergency laparotomy.

\section{Case report}

A 38-year-old woman was admitted with a 2-day history of severe, right lower abdominal pain.

The pain was colicky and associated with eight episodes of copious bilious vomiting. There was also progressive abdominal distension and obstipation. She had presented at a peripheral center 3 weeks before the index admission with a similar history, and was managed non-operatively for acute appendicitis with symptoms resolving within a few days post admission. Examination revealed a grossly distended abdomen with vague tenderness mainly on the right lower quadrant, with hyperactive bowel sounds. Digital rectal exam revealed an empty rectum with no masses. Complete blood count showed slightly elevated white cell count of $12 \times 109 / \mathrm{L}$. Abdominal knowledge) reported case of adult intussusception caused by ileal lipoma in Africa.

Key words: Intussusception, Ileal, Lipoma, Subserosal, Intestinal obstruction, Adult

Ann Afr Surg. 2019; 16(2):78-81

DOI:http://dx.doi.org/10.4314/aas.v16i2.8

Conflicts of Interest: None

Funding: None

(C) 2019 Author. This work is licensed under the Creative Commons Attribution 4.0 International License.

radiograph showed features of intestinal obstruction with multiple air fluid levels and paucity of rectal gas. Exploratory laparotomy revealed gross dilatation of the small bowel with telescoping of the distal ileum through the caecum and ascending colon up to the proximal transverse colon (Fig. 1).

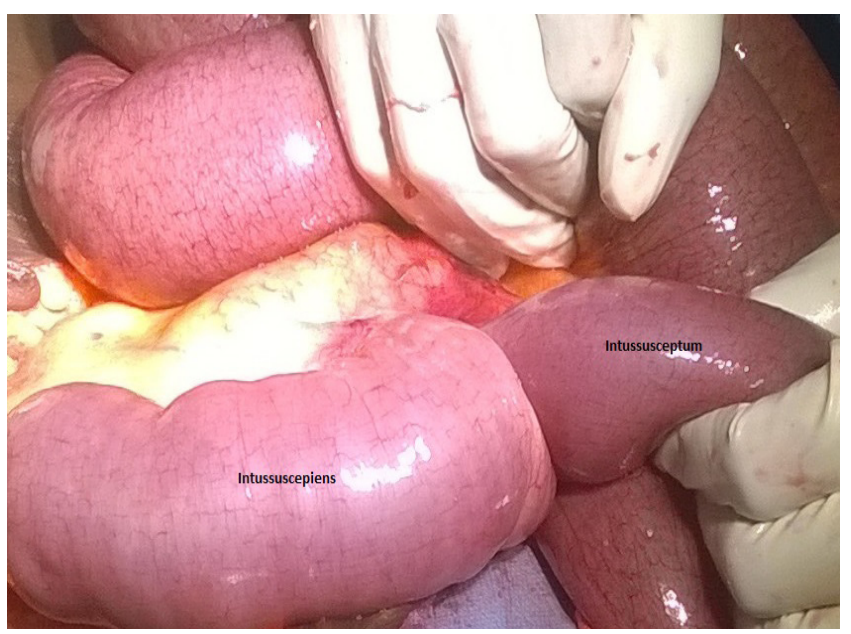

Figure 1. The intussuscepiens and intussusceptum

The dilated small bowel was manually reduced and a pathologic lead point was seen: a $5 \times 3 \mathrm{~cm}$ fatty mass located $30 \mathrm{~cm}$ from the ileocecal junction (Fig. 2).The segment was resected and anastomosed (Fig. 3) and the abdominal wound closed in layers. The patient made an uneventful recovery and was discharged on the 6th day postoperative. Histology revealed subserosal lipoma of the small bowel wall with no evidence of malignancy.

\section{Discussion}

Intussusception is defined as telescoping of a proximal segment of the gastrointestinal tract within the lumen of the adjacent segment. Although it is a common cause of intestinal 


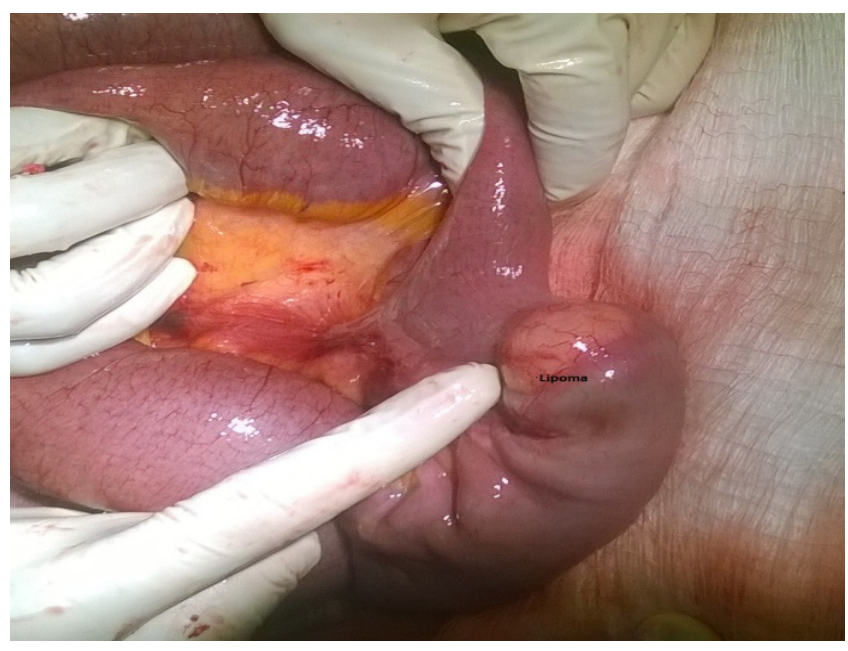

Figure 2. Intraoperative image showing the subserosal lipoma

obstruction in children, it is rare in adults. Adult intussusception represents $5 \%$ of all cases of intussusception and accounts for only $1-5 \%$ of intestinal obstructions in adults $(1,3)$. In contrast to intussusception occurring in children, adult intussusceptions have a pathologic lead point in up to $90 \%$ of cases (4).

Dean et al. classified intussusceptions into four types based on the location (7):

i. Enteroenteric: when confined to the small bowel

ii. Colocolic: involves the large bowel

iii. Ileocolic: when the terminal ileum telescopes into the ascending colon

iv. Ileocecal: originates from the ileocecal valve, usually difficult to differentiate from the ileocolic.

Intussusception is often misdiagnosed in adult patients and usually confused for more common pathologies such as complicated appendicitis or malignant processes. The index patient presented at a peripheral health facility in the early stage of the disease and was wrongly managed for acute appendicitis. A differential diagnosis of intussusception should always be considered in adult patients who present with acute onset intestinal obstruction.

Intestinal lipoma as a cause of intussusception is seen in only $6 \%$ of patients with intussusception (5). Apart from the often dramatic presentation with intestinal obstruction, intestinal lipomas may also present with chronic intestinal bleeding and diarrhea $(2,6)$. The average age at diagnosis was about 50 years, with slight female preponderance (8). Abdominal pain was the main presenting symptom in our patient. In a study done by Lindor et al. on 148 patients, the most common presentation of adult intussusception was abdominal pain seen in $72 \%$ of patients, next was nausea in $48 \%$ and vomiting in 36\%; $20 \%$ of patients in the series were asymptomatic (9).

The common triad seen in children - abdominal mass, bloody diarrhoea, abdominal pain - is rare in adults (10). Preoperative diagnosis of intussusception is difficult as patients are assumed to have other disease processes such as malignant obstruction, complicated appendicitis, enteritis or diverticulitis. Correct

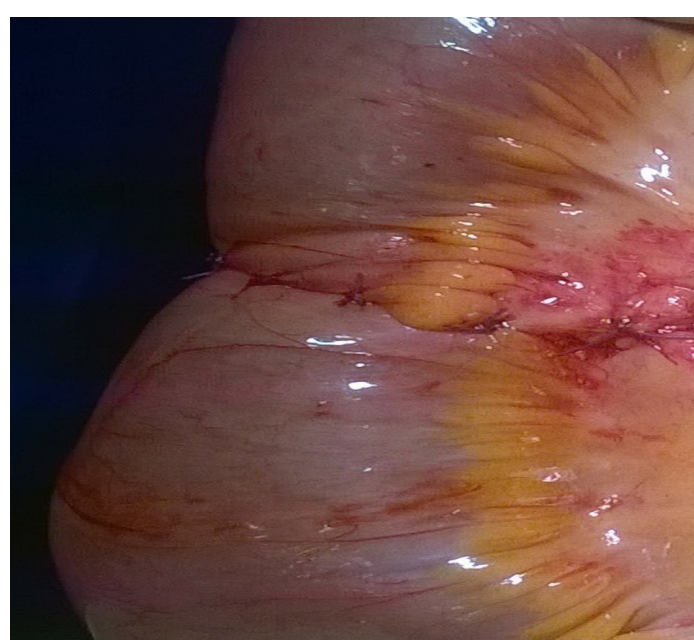

Figure 3. After resection and anastomosis

preoperative diagnosis is made about $40-50 \%$ of the time and requires a high index of suspicion (11). Gastrointestinal lipomas are rare; when present, they frequently occur at the terminal ileum and are the second most common benign tumors after gastrointestinal stromal tumors (12). Intestinal lipoma location may be submucosal, intramural or subserosal. The submucosa intestinal lipoma is the most common and is seen in about $90 \%$ of cases (13). Most gastrointestinal lipomas present as solitary masses, though in less than $10 \%$ of cases multiple lipomas may occur (14). Smaller lipomas $(<2 \mathrm{~cm})$ may not require intervention if they are asymptomatic. Larger lipomas $(>2 \mathrm{~cm})$ are more likely to be symptomatic and may require intervention.

Ileal intussusception can be diagnosed with endoscopy, barium enema, computed tomography (CT), and ultrasound. In endoscopy, ileal lipoma appears as a smooth yellow surface with a pedunculated or sessile base, or in either the "cushionsign" (poking the mass results in depression and subsequent restoration) or "naked fat sign" (ejection of fat during biopsy) (14). In sonography, intussusception usually appears as the classic doughnut sign or target sign or pseudo kidney sign. Ultrasonography may be limited by extensive bowel gas because of intestinal obstruction, and plain radiography may show a soft tissue mass, features of small bowel obstruction with paucity of colon feces, and gas (15). None of these signs are specific for intussusception. The presence of free air on plain radiograph is an ominous sign and signifies gut perforation.

Contrast-enhanced abdominal computed tomography (CT) is the diagnostic method of choice in adult intussusception, with diagnostic accuracy of up to $100 \%$. It may show the aspect of the bowel involved in the intussusception, which appears as target appearance, renieform or sausage appearance, and the presence or not of a lead point which has significance in the decision to intervene surgically. Ileal lipoma appears on CT as a well-marginated mass with fat attenuation (Hounsfield units between -80 and -120$)$. It can also reveal complications such as bowel gangrene and perforation. 
In a report by Kim et al., abdominal CT distinguished intussusception without a lead point (features: no signs of proximal bowel obstruction, target-like or sausage-shaped mass, layering effect) from that with a lead point (features: signs of bowel obstruction, bowel wall edema with loss of the classic three-layer appearance due to impaired mesenteric circulation, and demonstration of the lead mass) (4). This distinction may help reduce the number of unnecessary surgical interventions (16). All patients with a palpable abdominal mass, features of intestinal obstruction, gastrointestinal bleeding, or a lead point on CT should undergo operative exploration (8).

An abdominal CT would have been preferred for initial evaluation of the patient, but unavailability of funds limited the diagnostic work and only plain abdominal radiographs were taken. Complete intestinal obstruction, which the patient presented with, is an indication for laparotomy. Laparotomy is needed to set the intestinal obstruction to relieve the obstruction, and to confirm the diagnosis because usually several other differentials may be present. Compared with pediatric patients where intussusception is primary and benign, preoperative reduction with barium or air or water is not suggested as a definite treatment for adults (17).

Manual reduction is the gold standard treatment of pediatric intussusception, but it is rarely a method of treatment in adults except in patients with viable bowel, or when no lead point is identified by preoperative imaging, and in those at risk of short bowel syndrome, e.g. in Crohn's disease. Otherwise, resecting the lead point of the intussusception and histology are advised because as high as $40 \%$ of adult ileal intussusceptions have associated malignant pathologic lead points $(4,5)$. If a preoperative diagnosis of ileal lipoma has been made either via endoscopy or CT, and if the bowel is viable, manual reduction, segmental excision and simple repair will suffice. However, usually the exact diagnosis may not be known and many workers advocate resectioning the intussuscepted segment without reducing it, to prevent spillage and the tumor spreading if the lead point turns out to be malignant on histological examination (18). En-bloc resection reduces the possibility of recurrence and avoids repair/anastomosis on edematous, ischemic bowel. A formal oncologic resection in patients above 60 years with colonic intussusception is recommended because of the possible high incidence of a malignant lead point, which may approach $80 \%(4,19)$

The use of laparoscopy in the management of adult intussusception from ileal lipoma has been documented. Laparoscopy depends largely on proper patient selection and expertise of the surgeon. Laparoscopy rules out other differentials and provides a medium for possible intervention. Reduction and en-bloc resection and anastomosis can be performed laparoscopically (20).
Laparoscopy is still rudimentary in our setting; the index patient had a laparotomy with resection and anastomosis of the involved ileal segment.

\section{Conclusion}

Intussusception is a rare cause of intestinal obstruction in adults. A high index of suspicion is often required as preoperative diagnosis is infrequent without the use of investigative modalities such as the abdominal CT. Ileal lipoma as a cause of adult intussusception is uncommon. Manual reduction alone may not suffice and surgical excision of the involved segment is advised.

\section{References}

1. Lee DE, Choe JY. Ileocolic intussusception caused by a lipoma in an adult. World J Clin Cases. 2017; 5(6):254-7.

2. Laws HL, Aldrete JS. Small-bowel obstruction: A review of 465 cases. South Med J. 1976; 69(6):733-4.

3. Udo IA, Abudu EK, Uduma F. Adult intussusception: An 8-year institutional review. Niger Med J. 2016; 57(4):204-7.

4. Kim JW, Lee BH, Park SG, et al. Factors predicting malignancy in adult intussusception: An experience in university-affiliated hospitals. Asian J Surg. 2018; 41(1):92-7.

5. Azar T, Berger DL. Adult intussusception. Ann Surg. 1997; 226(2):134-8.

6. Kraniotis P, Pastromas G, Tsota I, et al. Giant ileocolic intussusception in an adult induced by a double ileal lipoma: A case report with pathologic correlation. Radiol Case Reports. 2016; 11(3):148-51.

7. Dean Dl, Ellis FH, Sauer WG. Intussusception in adults. AMA Arch Surg. 1956; 73(1):6-11.

8. Onkendi EO, Grotz TE, Murray JA, et al. Adult intussusception in the last 25 years of modern imaging: Is surgery still indicated? J Gastrointest Surg. 2011; 15(10):1699-1705.

9. Lindor RA, Bellolio MF, Sadosty AT, et al. Adult intussusception: Presentation, management, and outcomes of 148 patients. J Emerg Med. 2012; 43(1):1-6.

10. Marinis A, Yiallourou A, Samanides L, et al. Intussusception of the bowel in adults: A review. World J Gastroenterol. 2009; 15(4):407-11.

11. Eisen LK, Cunningham JD, Aufses AH. Intussusception in adults: Institutional review. J Am Coll Surg. 1999; 188(4):390-5.

12. Kurniawan N, Rüther C, Steinbrück I, et al. Tumours in the small bowel. Video GIE. 2014; 1(3):632-5.

13. Agarwal S, Pandey P, Singh S, et al. Colonic lipoma: A rare yet important cause of intestinal obstruction. Clin Cancer Investig J. 2016; 5(6):548-550.

14. Shehzad KN, Monib S, Ahmad OF, et al. Submucosal lipoma acting as a leading point for colo-colic intussusception in an adult. J Surg Case Reports. 2013; 2013(10).

15. White SJ, Biane CE. Intussusception: Additional observations on the plain radiograph. AJR Am J Roentgenol. 1982; 139:511-3.

16. Kim YH, Blake MA, Harisinghani MG, et al. Adult intestinal intussusception: CT appearances and identification of a causative lead point. Radiographics. 2006; 26(3):733-44.

17. Lianos G, Xeropotamos N, Bali C, et al. Adult bowel intussusception: Presentation, location, etiology, diagnosis and treatment. G Chir. 2013; 34:280-3. 
18. Balamoun H, Doughan S. Ileal lipoma-a rare cause of ileocolic intussusception in adults: Case report and literature review. World J Gastrointest Surg. 2011; 3(1):13-5.

19. Montiel-Jarquín AJ. Intussusception in adults: Surgical aspects. Can J Surg. 2007; 50(5):412.
20. Rupp S, Whitecar S, Ng P, et al. Laparoscopic resection of adult small bowel intussuscepting lipoma with intracorporeal anastomosis: A case report. Gastroenterol Hepatol Open Access. 2018; 9(1):23-5. 